DOI: http://dx.doi.org/10.20435/multi.v21i49.1092

\title{
Análise fitossociológica de um fragmento de cerrado em Campo Grande, MS
}

\section{Phytosociological analysis of a fragment of Cerrado in Campo Grande, MS}

\author{
Kwok Chiu Cheung ${ }^{1}$ \\ Letícia Koutchin-Reis ${ }^{2}$ \\ Carla Cristina Cerezoli de Jesus ${ }^{3}$
}

${ }^{1}$ Professor e coordenador do curso de Ciências Biológicas da UCDB. Mestre em Ecologia e Conservação pela Universidade Federal do Paraná (UFPR) e doutorando pela Rede Procentrooeste em Biotecnologia e Biodiversidade. E-mail: cheung@ucdb.br

${ }^{2}$ Graduanda do curso de Ciências Biológicas na Universidade Católica Dom Bosco, Campo Grande, MS. E-mail: leticiak.reis35@gmail.com

${ }^{3}$ Mestranda em Ecologia Vegetal na Universidade Federal de Mato Grosso do Sul, Campo Grande, MS. E-mail: cerezolijc@gmail.com 


\section{RESUMO ABSTRACT}

O Cerrado é detentor de imensa riqueza fisionômica e florística, com mais de 6.000 espécies fanerogâmicas registradas, possuindo uma das mais abrangentes floras dentre as savanas mundiais. 0 objetivo deste trabalho foi realizar um levantamento da composição florística em um fragmento do Cerrado e caracterizar suas fisionomias no Instituto São Vicente MS. 0 método utilizado foi amostragem das espécies de forma randômica, por toda a extensão da borda do fragmento e partes de seu interior. 0 critério de inclusão foi para espécies com hábito arbóreo, sub-arbóreo e arbustivo. No total foi possível registrar na área 40 famílias, com 94 espécies. Identificaram-se duas fisionomias que predominam na área (cerradão e mata ripária). 0 fragmento foi caracterizado por possuir áreas em estágio sucessional secundário, ou seja, ainda em processos de regeneração natural.

\section{PALAVRAS-CHAVE}

biodiversidade vegetal sucessão ecológica levantamento
Cerrado holds immense wealth physiognomy and floristic, with more than 6,000 registered phanerogams species, making it one of the most comprehensive floras among the world's savannas. The objective of this study was to survey the floristic composition in a cerrado fragment and characterize their faces at the Institute São Vicente MS. The sampling method was the recognition of species randomly, the length of the edge of the fragment and parts of its interior. The inclusion criterion was for species with arboreal habit, sub tree and shrub. It was possible to register in the area, 40 families, and 94 species. It sets up two faces that dominate the area (cerrado and riparian vegetation). The fragment is characterized by being in succession a secondary stage, or even natural processes of regeneration

\section{KEY WORDS}

plant biodiversity

ecological succession

survey 


\section{INTRODUÇÃo}

Cerca de dez por cento de todos os organismos do planeta encontra-se no Brasil, esse índice o torna um dos países com diversidade biológica elevadíssima (MYERS et al., 2000). Dentre tantas paisagens brasileiras, o Cerrado é um Bioma reconhecido como a savana mais rica do mundo em biodiversidade com presença de distintos ecossistemas (IBAMA, 2001).

Segundo Pivello (2003), o Cerrado é detentor de imensa riqueza fisionômica e florística, com mais de 6.000 espécies fanerogâmicas registradas, sendo assim uma das mais abrangentes floras dentre as savanas mundiais (MENDONÇA et al.,1998). Com uma paisagem descrita como mosaico vegetacional, que vai desde plantas lenhosas (árvores e arbustos) até herbáceas, torna-se, assim, uma região muito peculiar e muito diversificada fisionomicamente (RIBEIRO; WALTER, 1998).

No Estado de Mato Grosso do Sul, o Cerrado é a formação vegetacional predominante, e grande parte dessas áreas já não possui mais a cobertura vegetal original, estando atualmente ocupada por paisagens antrópicas (FELFILI et al., 2002).

A fitofisionomia mais comum é o cerrado stricto sensu, formação savânica caracterizada por possuir um estrato rasteiro bastante desenvolvido, constituído principalmente por gramíneas, e um estrato lenhoso não muito denso, em que as copas das árvores não formam um dossel contínuo (RIBEIRO; WALTER, 1998).

Outra fitofisionomia é a mata ciliar, uma das formações vegetais mais importantes, pois serve de proteção aos rios e córregos, formando uma comunidade de plantas, animais e outros organismos vivos que interagem com outros componentes não vivos, como os próprios rios (NASS, 2009).

A mata ciliar apresenta espécies arbóreas caducifólias que conferem aspecto geral de mata semidecídua. Florísticamente, as espécies que a habitam a tornam similar à mata seca, entretanto 
matas ciliares possuem estrutura mais densa e mais alta (NICÁCIO, 2001), formando então a copa e cobertura dos rios.

Atualmente, a expansão das fronteiras agrícolas, aliada ao baixo percentual de áreas protegidas por unidades de conservação de uso restrito, provocou uma redução drástica na área do Cerrado brasileiro, estimada em aproximadamente $37 \%$ da cobertura original (FELFILI et al., 2002). A falta de informações a respeito da diversidade e conservação em áreas de "tensão ecológica" indica o baixo índice de pesquisas sobre a composição florística, a fitossociologia e a similaridade dos tipos vegetacionais e de seu entorno (IBGE, 1993; ARAÚJO, 2008).

Esses baixos índices não são diferentes quando se trata da distribuição e organização da biodiversidade nas comunidades do Cerrado, tais pesquisas podem trazer informações de grande importância para avaliar os impactos antrópicos, planejar a criação de unidades de conservação e a adotar de técnicas de manejo (FELFILI; SILVA JÚNIOR, 2001). Levantamentos florísticos são importantes para o conhecimento da biodiversidade de determinada região e são escassos em relação ao número vegetacional existente no estado (KOCHANOVSKI et al., 2008). Segundo Felfili et al. (2002), apenas $0,5 \%$ da sua área total está protegida por unidades de conservação de uso restrito, e 3,6\% protegidos por alguma categoria de unidade de conservação.

Os primeiros trabalhos relativos aos aspectos fisionômico-florísticos do cerradão tratam da observação e da descrição das principais características estruturais (altura, densidade, estratificação etc.) bem como da composição geral das espécies, inclusive indicando as espécies mais abundantes e com portes mais elevados em diferentes fitofisionomias (SÓLORZANO et al., 2011).

Dentro desse contexto, o objetivo geral deste trabalho foi analisar e registrar a fitossociologia de um fragmento do Cerrado no Instituto São Vicente-MS, tendo como intuito levantar as espécies encontradas; caracterizar as fitofisionomias presentes na 
área; identificar qual o estágio de sucessão ecológica é predominante e qual o índice de diversidade nas áreas desse fragmento.

\section{MATERIAIS E MÉTODOS}

\section{Área de estudo}

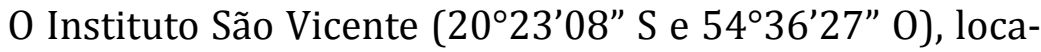
lizado no município do Campo Grande, possui uma área total de 191 hectares, sendo 20 ha de reserva legal, está situado na região da Lagoa da Cruz, em Campo Grande, MS (Figura 1). 0 local é destinado à realização de pesquisas em diversas áreas de ensino - biológicas, saúde e educação ambiental - proporcionando a vivência acadêmica dos estudantes em suas áreas de formação.

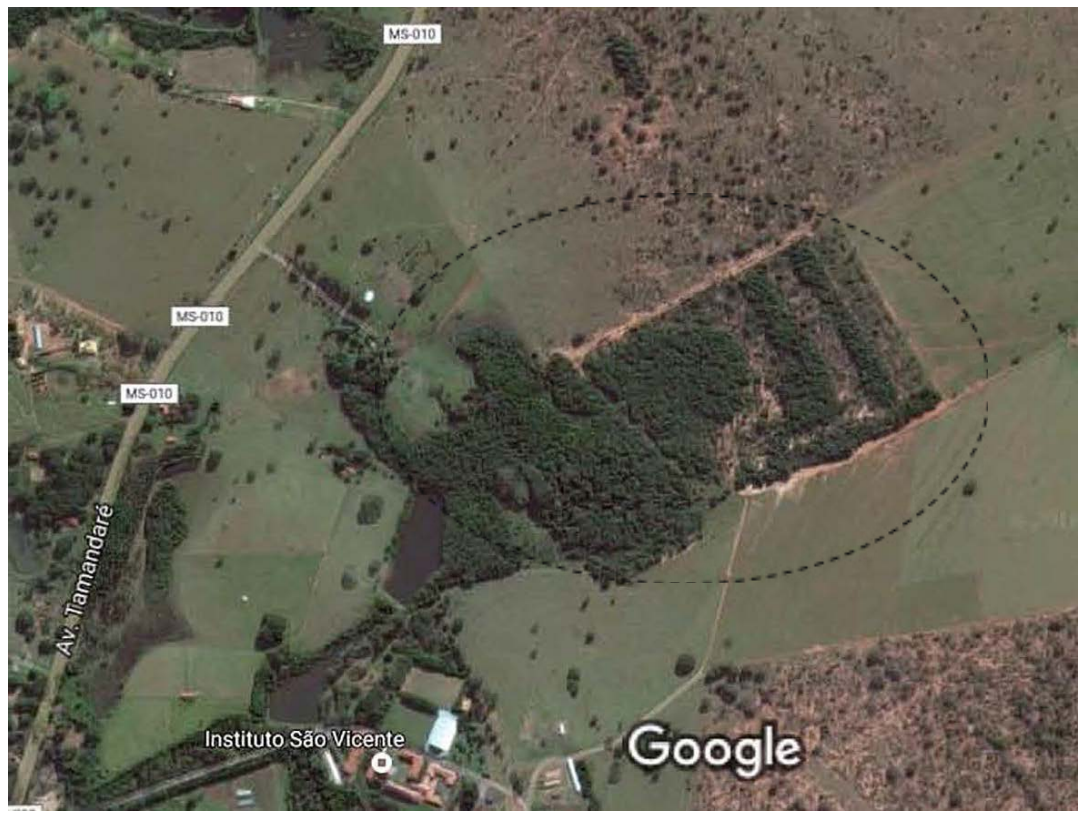

Figura 1 - Fragmento de Cerrado onde foram realizadas as coletas florísticas. Instituto São Vicente, Lagoa da Cruz, Fazenda Escola. Fonte: Google Maps, 2016 
Atualmente o local, considerado um fragmento de Cerrado, está em processo avançado de regeneração, porém, no passado, sofreu forte intervenção humana para criação de gado, agricultura e exploração da madeira. A área de estudo foi dividida em dois grandes pontos, caracterizados por uma área onde há uma nascente e uma área de afloramento do lençol freático, dominada por arbustos lacustres de pequeno porte e gramíneas, onde foram feitas as coletas de material botânico, inéditas até o momento (MENDONÇA; DANNI-OLIVEIRA, 2007).

\section{Método de coleta}

O método de amostragem para o reconhecimento das espécies foi realizado de forma randômica, no qual se percorreu a pé toda a extensão da borda do fragmento e partes de seu interior, sendo observado todos os aspectos morfológicos disponíveis nas plantas, tais como ramos, folhas, flores, frutos, ritidoma, casca interna, exsudatos, resina, cheiro, estípulas, dentre outras. A identificação das plantas teve como base nomenclatura especializada segundo o sistema Angiosperm Phylogeny Group (APG) de 2009. Para as plantas não identificadas em campo, foram montadas exsicatas em prensas de madeira com papelão e jornal embebidos em álcool e posteriormente secas em estufa, visando à conservação do material coletado.

\section{Análise de dados}

Para a confirmação das exsicatas, utilizamos bibliografia especializada de herbários virtuais do Missouri Botanical Garden e Neotropical Herbarium Specimens. Para a suficiência amostral, foi calculada com base na curva espécie-área ou curva de acumulação de espécies. Para o cálculo de riqueza e abundância foi aplicado ao índice de diversidade de Shannon-Winer (WHITTAKER; JONES, 1994): $\mathbf{H}^{\prime}=-\boldsymbol{\Sigma}$ pi. Ln pi, com objetivo de correlacionar 
ao de diversidade nas áreas amostradas com suas fitofisionomias encontradas em espécies, famílias encontradas e correlação ao estágio de regeneração estacional da área.

\section{RESULTADOS E DISCUSSÕES}

Foram levantadas e amostradas cerca de 40 famílias, com 94 espécies diferentes de hábito arbóreo e arbustivo no fragmento do Cerrado. Com base na análise das fisionomias da área de estudo, foi possível definir as fitofisionomias presentes no fragmento, que são definidas por duas fisionomias que predominam a área. Essas fisionomias vão de formações florestais, que inclui cerradão, à mata ripária, com características de mata ciliar e matas de galerias.

Nesse contexto, as espécies registradas que caracterizam essas fitofisionomias são, por exemplo, espécies da família Fabaceae, cujo hábito, maioria arbóreo, predominante em áreas mais secas como cerradão, e espécies da família Melastomataceae, que caracterizam a mata riparia, como hábito arbustivo predominante (Tabela 1).

Tabela 1 - Espécies registradas no Instituto São Vicente, Lagoa da Cruz (UCDB) no período de um ano (2014-2016), separadas por família/espécie e nome popular; Para L (Local registado), Tse (Tipo de sucessão ecológica da espécie no local), NI (Espécie não identificada).

\begin{tabular}{|c|c|c|c|}
\hline FAMÍLIA / ESPÉCIE & NOME POPULAR & L/Tse & Hábito \\
\hline Anacardiaceae & & & \\
\hline Anacardium sp. L. & Caju & $\mathrm{C} / \mathrm{P}$ & Sub arbóreo \\
\hline Tapirira guianensis Aubl. & Tapirirá & $\mathrm{C} / \mathrm{P}$ & Arbóreo \\
\hline \multicolumn{4}{|l|}{ Annonaceae } \\
\hline Anona coriaceae Mart. & Fruta do conde & $\mathrm{C} / \mathrm{P}$ & Sub arbóreo \\
\hline Anona monticola Mart. & Marolo & $\mathrm{C} / \mathrm{P}$ & Sub arbóreo \\
\hline Duguetia furfuracea (A. St.-Hil.) Saff & & $\mathrm{C} / \mathrm{P}$ & Arbusto \\
\hline Unonopsis lindmanii R.E. Fr. & & $\mathrm{M} / \mathrm{P}$ & Arbóreo \\
\hline Xylopia aromatica Lam. E Mart. & Pimenta de macaco & $\mathrm{C} ; \mathrm{M} / \mathrm{P}$ & Arbóreo \\
\hline Anona sp. Mill. & Marolo & $\mathrm{C} ; \mathrm{M} / \mathrm{P}$ & Sub arbóreo \\
\hline
\end{tabular}




\section{FAMÍLIA / ESPÉCIE}

\section{NOME POPULAR L/Tse}

Hábito

Xylopia sp. L.

C

Arbóreo

\section{Apocynaceae}

Himatanthus obovatus Müll. Arg. Lírio do campo

C/P Arbóreo

\section{Asteraceae}

Achyrocline alata (Kunth) DC.

C/M Arbusto

Vernonia polysphaera Baker

$\mathrm{C} / \mathrm{M} \quad$ Arbusto

Piptocarpha sp. (Sch.Bip) Cabrera. Vassourão

C/P Sub arbóreo

\section{Bignoniaceae}

Arrabidaea florida Dc.

M/P Sub arbóreo

Jacaranda rufa S.M

$\mathrm{C} / \mathrm{P} \quad$ Arbusto

NI

C Sub arbóreo

Fridericia platyphylla L.G. Lohmann

C/P Sub arbóreo

NI

C/P Arbóreo

\section{Caesalpinaceae}

Copaifera langsdorffii Desf.

Copaíba

C/NP Arbóreo

Calliandra mucugeana Renvoize.

Caliandra

C/P Arbóreo

\section{Cannabaceae}

Trema micrantha (L.) Blume.

Pau pólvora

C;M/P Arbóreo

\section{Caryocaceae}

Caryocar brasiliense Camb.

Pequi

C/P Arbóreo

\section{Celastraceae}

Plenckia populnea Reissek.

Marmelo

C;M/P Sub arbóreo

salacia crassifolia Mart.

Bacupari do cerrado

$\mathrm{C} / \mathrm{P}$ Sub arbóreo

\section{Chrysobalanaceae}

Hirtella gracilipes (Hook.f.) Prance. Irtela

C/P Arbóreo

Clusiaceae

Calophyllum brasiliense Cambess.

Guanandi

M/P Arbóreo

\section{Connaraceae}

Connarus suberosus Planch.

Cascudinho

C/P Arbóreo

Rourea induta Planch.

Pau-de-Porco

C/P Sub arbóreo

\section{Dilleniaceae}

Curatella americana L.

Lixeira

$\mathrm{C} / \mathrm{P}$

Arbóreo

NI

C Sub arbóreo

\section{Euphorbiaceae}

Maprounea guianensis Aubl.

Vaquinha

Croton urucurana Baill.

Mabea fistulifera Mart.

\section{Fabaceae}

Dipteryx alata Vogel.

Sangra d'água

$\mathrm{C} ; \mathrm{M} / \mathrm{P}$

$\mathrm{C} / \mathrm{NP} \quad$ Arbóreo

Canudo de pito

$\mathrm{C} / \mathrm{P}$

Arbóreo

Inga laurina (Sw.) Willd.

Bauhinia forficata Benth.

Leptolobium dasycarpum Vogel.
Baru

Ingá

$\mathrm{C} / \mathrm{NP}$

$\mathrm{C} / \mathrm{NP}$

Pata de vaca

Chapada
$\mathrm{C} / \mathrm{P}$

$\mathrm{C} / \mathrm{P}$
Arbóreo

Arbóreo

Arbóreo

Arbóreo 


\section{FAMÍLIA / ESPÉCIE}

\section{NOME POPULAR L/Tse}

\section{Hábito}

Stryphnodendron adstringens (Mart.) Coville

Barbatimão

C/NP Arbóreo

Hymenaea stigonocarpa Mart.

Jatobá C/NP Arbóreo

Dalbergia miscolobium Benth.

Caviúna

C/NP Arbóreo

Andria sp.

Pterodon emarginatus Vogel.

C;M/P Arbóreo

Anadenanthera colubrina Vellozo.

Sucupira

$\mathrm{C} / \mathrm{P}$

Arbóreo

Flacourticeae

Casearia rupestris Eichler.

Angico

C/NP Arbóreo

\section{Icacinaceae}

Emmotum nitens Benth.

Guaçatunga

C/P Sub arbóreo

\section{Lauraceae}

Ocotea nectandrifolia Mez.

Carvalho

C/P Árbóreo

\section{Loganiaceae}

Strychnos pseudoquina A. St. Hil.

\section{Malpighiaceae}

Byrsonima intermedia A. Juss.

Byrsonima verbascifolia L. Dc.

\section{Melastomataceae}

Tococa guianensis Aubl.

Canela

C/P Arbóreo

Miconia burchelli Triana.

Quina

$\mathrm{C} / \mathrm{NP}$

Arbóreo

Microlicia myrtoideaCham.

Miconia nervosa var. mediana J.F.

Macbr.

Trembleya phlogiformis DC.

miconia albicans(Sw.) Steud.

Murici

$\mathrm{C} / \mathrm{P} \quad$ Arbusto

Murici branco

$\mathrm{C} / \mathrm{P} \quad$ Arbusto

Mouriri ellipticaMart.

$\mathrm{M} / \mathrm{P} \quad$ Arbusto

$\mathrm{M} / \mathrm{P} \quad$ Arbusto

$\mathrm{M} / \mathrm{P} \quad$ Arbusto

Meliaceae

Guarea Guidonea (L.) Sleumer.

Camboatá

C;M/P Sub arbóreo

Trichilia hirta L.

Catiguá-arco-depeneira

$\mathrm{M} / \mathrm{P} \quad$ Arbusto

$\mathrm{M} / \mathrm{P} \quad$ Arbusto

C;M/NP Sub arbóreo

\section{Miristicaceae}

Virola sebifera Aubl.

Pau de sebo

C/P Arbóreo

Moraceae

Brosimum gaudichaudii Trecul.

Mama cadela

$\mathrm{C} / \mathrm{P} \quad$ Arbusto

Ficus obtusifolia Kunth.

Figueira

C;M/NP Arbóreo

Ficus sp. L.

Figueira

C;M/NP Arbóreo

Nyctaginaceae

Guapira sp. Aubl.

Guapira

C;M/P Arbóreo

Papilionoideae

Machaerium vestitum Vogel.

Jacarandá branco

$\mathrm{C} / \mathrm{NP}$

Arbóreo 


\section{FAMÍLIA / ESPÉCIE}

\section{NOME POPULAR L/Tse}

\section{Hábito}

Phyllanthaceae

Hyeronima alchorneoides Allemão. Folha de bordo C/P Arbóreo

Piperaceae

Piper aduncumAubl.

M/P Sub arbóreo

Piper arboreum Aubl.

Pariparoba

C;M/P Sub arbóreo

Primulaceae

Myrsine coriaceae (Sw.) R.Br.

Capororoca

C;M/P Sub arbóreo

Proteaceae

Roupala brasiliensis Klotzsch.

Carvalho brasileiro

C/NP

Arbóreo

Rhamnaceae

Ziziphus joazeiro Mart.

Joazeiro

C/NP Arbóreo

\section{Rosaceae}

prunus sellowii Koehne.

Pessegueiro bravo C/NP Arbóreo

\section{Rubiaceae}

Coccocypselum lanceolatum (Ruiz \&

Pav.) Pers.

CM/P Arbóreo

Faramea spathacea Müll. Arg. ex

Standl

M/P Arbóreo

Tocoyena formosa Cham. \& Schltdl. Guamarú

$\mathrm{C} / \mathrm{P} \quad$ Sub arbóreo

Rudgea viburnoides (Cham.) Benth. Congonha de bugre

C/P Arbóreo

Palicourea rigida H.B.K.

Bate-caixa

$\mathrm{C} / \mathrm{P}$ Sub arbóreo

Psychotria carthagenensis Jacq.

Sapindaceae

Matayba elaeagnoides Radlk.

Carne de vaca

C/P Arbóreo

\section{Sapotaceae}

Chrysophyllum gonocarpum (Mart. \&

Eichler) Engl.

Aguaí $\quad$ C/P Sub arbóreo

\section{Simaroubaceae}

Simarouba versicolor St. Hil.

Perdiz

$\mathrm{C} / \mathrm{P}$

Arbóreo

\section{Siparunaceae}

Siparuna guianensis Aublet.

Negramina

CM/P Arbóreo

\section{Solanaceae}

Solanum grandiflorum J.F. Macbr.

C;M/P Abusto

\section{Urticaceae}

Embaúba

M/P Sub arbóreo

Cecropia pachystachya Trec.

Vochysiaceae

Vochysia haenkeana Mart.

Vochysia tucanorum Mart.

Qualea grandiflora Mart.

$\begin{array}{lll}\text { Cambará } & \mathrm{C} / \mathrm{NP} & \text { Arbóreo } \\ \text { Cinzeiro } & \mathrm{C} / \mathrm{NP} & \text { Arbóreo } \\ \begin{array}{l}\text { Pau terra da folha } \\ \text { larga }\end{array} & \mathrm{C} / \mathrm{NP} & \text { Arbóreo } \\ \begin{array}{l}\text { Pau terra da folha } \\ \text { pequena }\end{array} & \mathrm{C} / \mathrm{NP} & \text { Arbóreo }\end{array}$




\section{FAMÍLIA / ESPÉCIE}

Qualea multiflora Mart.

NI

NI

NI

$\mathrm{NI}$

NI

$\mathrm{NI}$

NI

NI

NI

NI

\section{NOME POPULAR L/Tse}

$\mathrm{M}$

$\mathrm{C} ; \mathrm{M}$

$\mathrm{C} ; \mathrm{M}$

C

C

C Sub arbóreo

$\mathrm{C} ; \mathrm{M}$

C

C

C
Hábito

Sub arbóreo

Sub arbóreo

Arbusto

Arbusto

Sub arbóreo

Arbusto

Arbusto

Arbóreo

Arbusto

Arbusto

Segundo Ribeiro e Walter (1998), o cerradão é uma formação florestal com aspectos xeromórficos (resistência à seca), tendo sido conhecido pelo nome "Floresta Xeromorfa", tipificado como sendo "uma mata mais rala e fraca". Caracteriza-se pela presença de espécies que ocorrem no cerrado sentido restrito e também por espécies de mata, apresentando dossel (copa) predominantemente contínuo, e sua cobertura arbórea, que pode oscilar entre 50 a 90\%, com altura média do estrato arbóreo, varia de 8 a 15 metros, proporcionando condições de luminosidade que favorecem a formação de estratos arbustivos e herbáceos diferenciados.

Já a outra fitofisionomia encontrada, em sua definição, mata ripária pode ser subdividida em duas categorias: mata ciliar e mata de galeria. A mata ciliar é definida como a vegetação florestal que acompanha os rios de médio e grande porte na região do Cerrado, em que a vegetação arbórea não forma galerias. Em geral essa mata é relativamente estreita em ambas as margens, dificilmente ultrapassando 100 metros de largura em cada. É comum a largura em cada margem ser proporcional à do leito do rio, embora em áreas planas a largura possa ser maior. Porém a mata ciliar ocorre geralmente sobre terrenos acidentados, podendo haver uma transição nem sempre evidente para outras fisionomias florestais como a mata seca e o cerradão (ICMBio, 2014). 
A distribuição das espécies encontradas em relação à diversidade nos fragmentos e ao estágio de sucessão ecológica, mostrada na (Tabela 2) pelo índice de Shannon, foi definida pela riqueza de famílias encontradas nas fitofisionomias e a abundância de espécies encontradas em cada fitofisionomia dessas famílias e, em outra divisão, apresenta a riqueza e abundância de espécies encontradas de pioneiras e clímax no fragmento inteiro avaliado. Isso mostra que existe uma predominância na abundância e riqueza de espécies pioneiras sobre as espécies clímax, quando tratando da área total do fragmento. Essa relação pode estar ligada ao fator de regeneração pelo qual a área passa. Chazdon (2008) comenta que a elevada densidade de espécies pertencentes a grupos sucessionais iniciais é uma característica peculiar de florestas naturais ou antropicamente perturbadas, visto que, em florestas tropicais maduras, tais grupos tendem a ocorrer em baixas densidades (raras), vinculadas a clareiras ou bordas naturais (ecótonos). Dessa forma, florestas em fases iniciais de regeneração apresentam elevada abundância de espécies pioneiras, principalmente porque estas exercem função "cicatrizadora" e são as primeiras a predominarem no ambiente, devido ao seu rápido crescimento e acúmulo de biomassa (FONSECA; CARVALHO, 2012).

Tabela 2 - Distribuição correlativa entre as famílias e espécies nos diferentes tipos de fitofisionomias encontradas na área, contando com a riqueza e abundância, com índice de Shannon H', Equitabilidade - Equitability_J e Variância - Variance.

\begin{tabular}{lcccc}
\hline & \multicolumn{2}{c}{ Fitofisionomias } & \multicolumn{2}{c}{ Área Total do Fragmento } \\
\hline & Cerradão & Mata Ripária & Espécies Pioneiras & Espécies Clímax \\
\hline Riqueza & 40 & 39 & 35 & 12 \\
Abundância & 76 & 28 & 63 & 21 \\
Shannon H' & 0,3358 & 0,2633 & 0,3271 & 0,2025 \\
Equitability_J & 0,9233 & 0,9292 & 0,9275 & 0,8864 \\
Variance & 0,011395 & 0,02681 & 0,012877 & 0,042001 \\
\hline
\end{tabular}


A abundância verificada separadamente em ambas as fitofisionomias distintas mostrou que essa percentagem não apresenta uma diferença considerável. Em relação à riqueza, as percentagens destoam consideravelmente. Essa diferença na riqueza relacionada à fitofisionomia pode estar ligada com o mecanismo de dispersão utilizado por essas espécies, ou com o banco de plântulas e sementes da área, visto que no passado era, entre outras coisas, área de pastagem.

A equitabilidade e variância que define a uniformidade e a padronização dessa da riqueza e abundância de uma vegetação, percebe-se que, no fragmento avaliado, existe uma uniformidade alta na área total, ou seja, nas duas fitofisionomias caracterizadas há uma diversidade ainda baixa e pouco variável, que é verificada pelo seu estágio de regeneração, já que o índice de variância e equitabilidade do fragmento de espécies clímax foi menor do que espécies pioneiras. Esse padrão é normalmente encontrado em florestas tropicais urbanas e tem como uma das suas características o comprometimento da riqueza de espécies (FONSECA; CARVALHO, 2012).

Segundo Daubenmire (1968), uma ferramenta utilizada para a compreensão da sucessão é a distribuição diamétrica, ou seja, a determinação da idade das árvores é realizada através da contagem dos anéis de crescimento. Porém, nos sistemas tropicais, como o bioma Cerrado, esse método torna-se impraticável uma vez que os anéis não são anuais, não sendo sua periodicidade bem definida. Nesse caso, para avaliar as idades das árvores, utiliza-se a medição dos diâmetros, esperando que reflita a estrutura de tamanho das populações (HARPER, 1977).

A característica fisionômica dessas espécies é um fator importante para determinação em estágios de sucessão ecológica, ocorrendo no fragmento cerca de $73 \%$ de espécies pioneiras (35\% primárias e $38 \%$ secundárias) e cerca de 31\% de espécies clímax. Isso determina o estágio de sucessão e mostra que essa 
área ainda está em amplo processo de regeneração, por ser a diversidade de espécies ainda alta, contudo a área apresenta uma maior porcentagem de espécies de primeiro estágio, pioneiras. Já a pequena porcentagem de espécies com características de estágio de sucessão avançada, clímax, representada por árvores maiores e mais antigas, tem uma taxa de reprodução mais baixa, justificando assim uma abundância baixa. Na área estudada há presença de espécies de lianas que recobrem o fragmento, facilitando então o encontro das copas das grandes árvores.

\section{REFERÊNCIAS}

ARAÚJO, R. A. Florística e estrutura da Comunidade Arbórea em Fragmento Florestal Urbano no Município de Sinop, Mato Grosso. 2008. 132f. Dissertação (Mestrado em Ciências Florestais e Ambientais) Universidade Federal de Mato Grosso, Cuiabá, MT, 2008

CHAZDON, R. L. Chance and determinism in tropical forest sucession. In: CARSON, W. P.; SCHNITZER, S. A. (Ed.). Tropical Forest Community Ecology. Chichester: Blackwell Publishing Ltd, 2008. p. 384-408.

DAUBENMIRE, R. Plant communities: a textbook of plant synecology. New York: Harper e Row, 1968.

FELFILI, J. M.; NOGUEIRA, P. E. JÚNIOR, M. C. S.; MARIMON, B. S.; DELITTI, W. B. C. Composição florística e fitossociologia de um cerrado sentido restrito no município de Água Boa-MT. Acta Botanica Brasílica, São Paulo, v. 16, n. 1, p. 103-112, 2002.

FELFILI, J. M.; SILVA JÚNIOR, M. C. Biogeografia do bioma cerrado: estudo fitofisionômico na Chapada do Espigão Mestre do São Francisco. Universidade de Brasília, Faculdade de Tecnologia, Departamento de Engenharia Florestal, Brasília, 2001.

FONSECA, C. R.; CARVALHO, F. A. Aspectos florísticos e fitossociológicos da comunidade arbórea de um fragmento urbano de floresta atlântica (Juiz de Fora, MG, Brasil). Biosci. J., Uberlândia, v. 28, n. 5, p. 820-832. set./out. 2012.

GOOGLE MAPS. Instituto São Vicente, Campo Grande, MS, 2016. 
HARPER, J. L. Population biology of plants. London: Academic, 1977. ICMBio. Fitofisionomias - Mata Ripária. Projeto Corredor Ecológico Região do Jalapão, 2014. Disponível em: <http://www.icmbio.gov.br/ projetojalapao/pt/biodiversidade-3/fitofisionomias.html?start=6> . Acesso em: 29 maio 2016.

INSTITUTO BRASILEIRO DE GEOGRAFIA E ESTATÍSTICA (IBGE). Centro de Documentação e Disseminação de Informações. Normas de apresentação tabular. Fundação Instituto Brasileiro de Geografia e Estatística, Centro de Documentação e Disseminação de Informações. 3. ed. Rio de Janeiro: IBGE, 1993. 62p.

INSTITUTO BRASILEIRO DO MEIO AMBIENTE E DOS RECURSOS NATURAIS RENOVÁVEIS (IBAMA). Ecossistemas brasileiros. Brasília: Edições IBAMA, 2001. 49p.

KOCHANOVSKI, F. J.; SCIAMARELLI, A.; DUARTE, C. U. N. B. D. Levantamento florístico da vegetação herbácea e subarbustiva do córrego mamangaba, Mundo Novo, Mato Grosso do Sul, Brasil. In: SIMPÓSIO NACIONAL CERRADO. DESAFIO ESTRATÉGIAS PARA O EQUILÍBRIO ENTRE SOCIEDADE, AGRONEGÓCIO E RECURSOS NATURAIS, XV., 2008. Brasília, DF. Anais..., Brasília, DF: EMBRAPA CERRADOS. 2008.

MENDONÇA, F.; DANNI-OLIVEIRA, I. M. Climatologia: noções básicas e climas do Brasil. São Paulo: Oficina de textos, 2007.

MENDONÇA, R.; FELFILI, J. M.; WALTER, B. M. T.; SILVA JÚNIOR, M. C.; REZENDE, A. V.; FILGUEIRAS, T. S.; NOGUEIRA, P. E. N. Flora vascular do Cerrado. In: SANO, S. M.; ALMEIDA, S. P. (Ed.). Cerrado: ambiente e flora. Brasília, DF: Embrapa, p. 287-556, 1998.

MYERS, N.; MITTERMEIER, R. A.; MITTERMEIER, C. G.; FONSECA, G. A. B.; KENT, J. Biodiversity hotspots for conservation priorities. Nature, v. 403, p. 853-858, 2000.

NASS, P. D. Mata ciliar - o corredor da natureza: a mata que cerca os rios é fundamental na preservação do ambiente e do equilíbrio ecológico. Cultura Secular, Brasília, DF, v. 7, n. 13, jan./jun. 2009. Disponível em: <http://cultura.secular.com.br/13-mai2009/mataciliar.html>. Acesso em: 2 jun. 2016. 
NICÁCIO, M. E. J. A manutenção de mata ciliar: um ativo permanente. Revista de estudos sociais, Cuiabá, ano 3, n. 6, p. 85-92, 2001.

PIVELLO, V. R. Estudos para a conservação dos recursos biológicos do Cerrado - o exemplo da “Gleba Cerrado Pé-de-Gigante" (Parque Estadual de Vassununga, Santa Rita do Passa Quatro, SP). 2003. 110f. Tese (LivreDocência) - Instituto de Biociências, Universidade de São Paulo, São Paulo, SP, 2003.

RIBEIRO, J. F.; WALTER, B. M. T. Fitofisionomias do bioma Cerrado. In: SANO, S. M.; ALMEIDA, S. P. (Ed.). Cerrado: ambiente e flora. Planaltina: Embrapa/CPAC, 1998. p. 89-116.

SÓLORZANO, A.; PINTO, J. R. R; FELFILI, J. M.; HAY, J. D. V. Perfil florístico e estrutural do componente lenhoso em seis áreas de cerradão ao longo do bioma Cerrado. Acta Botanica Brasilica, Belo Horizonte, v. 26, n. 2, p. 328-341, 2011.

WHITTAKER, R. J.; JONES, S. H. The role of frugivorous bats and birds in the rebuilding of a tropical forest ecosystem, Krakatau, Indonesia. Journal of Biogeography, v. 21, n. 3, p. 245-258, maio 1994. 